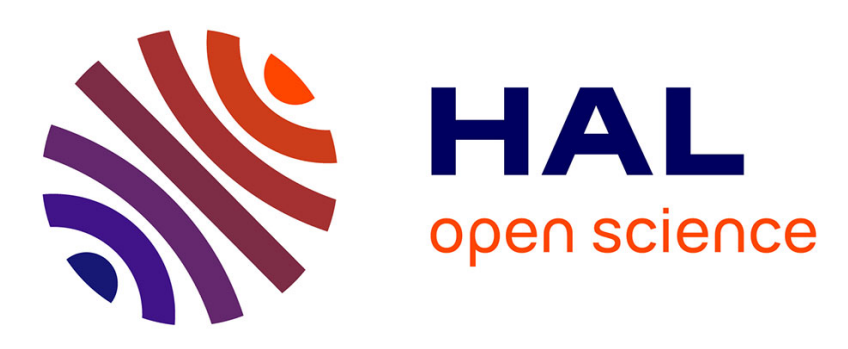

\title{
Beyond Mouse and Keyboard: Expanding Design Considerations for Information Visualization Interactions
}

Bongshin Lee, Petra Isenberg, Nathalie Henry Riche, Sheelagh Carpendale

\section{- To cite this version:}

Bongshin Lee, Petra Isenberg, Nathalie Henry Riche, Sheelagh Carpendale. Beyond Mouse and Keyboard: Expanding Design Considerations for Information Visualization Interactions. IEEE Transactions on Visualization and Computer Graphics, 2012, Proceedings Scientific Visualization / Information Visualization, 18 (12), pp.2689-2698. 10.1109/TVCG.2012.204 hal-00717203

\section{HAL Id: hal-00717203 \\ https://hal.inria.fr/hal-00717203}

Submitted on 25 Jan 2013

HAL is a multi-disciplinary open access archive for the deposit and dissemination of scientific research documents, whether they are published or not. The documents may come from teaching and research institutions in France or abroad, or from public or private research centers.
L'archive ouverte pluridisciplinaire HAL, est destinée au dépôt et à la diffusion de documents scientifiques de niveau recherche, publiés ou non, émanant des établissements d'enseignement et de recherche français ou étrangers, des laboratoires publics ou privés. 


\title{
Beyond Mouse and Keyboard: Expanding Design Considerations for Information Visualization Interactions
}

\author{
Bongshin Lee, Petra Isenberg, Nathalie Henry Riche, and Sheelagh Carpendale
}

\begin{abstract}
The importance of interaction to Information Visualization (InfoVis) and, in particular, of the interplay between interactivity and cognition is widely recognized [12][15][32][55][70]. This interplay, combined with the demands from increasingly large and complex datasets, is driving the increased significance of interaction in InfoVis. In parallel, there have been rapid advances in many facets of interaction technologies. However, InfoVis interactions have yet to take full advantage of these new possibilities in interaction technologies, as they largely still employ the traditional desktop, mouse, and keyboard setup of WIMP (Windows, Icons, Menus, and a Pointer) interfaces. In this paper, we reflect more broadly about the role of more "natural" interactions for InfoVis and provide opportunities for future research. We discuss and relate general $\mathrm{HCl}$ interaction models to existing InfoVis interaction classifications by looking at interactions from a novel angle, taking into account the entire spectrum of interactions. Our discussion of InfoVis-specific interaction design considerations helps us identify a series of underexplored attributes of interaction that can lead to new, more "natural," interaction techniques for InfoVis.
\end{abstract}

Index Terms-Design considerations, interaction, post-WIMP, NUI (Natural User Interface).

\section{INTRODUCTION}

The importance of interaction to Information Visualization (InfoVis) and, in particular, of the interplay between interactivity and cognition is widely recognized [12][15][32][55][70]. Interaction particularly rises in significance in InfoVis as we engage with increasingly large and complex datasets. Many tasks on visualized data can no longer be completed using static images alone. As a consequence, information workers request more and more interactive data analysis instruments [18]. In addition, a general audience is beginning to create visualizations using data of their own interest [49][72][80]. The creation of visualizations and the exploration of data have a number of dedicated underlying interaction requirements causing challenges that visualization research is just beginning to address.

In parallel, but independently of the proliferation of InfoVis, interface and interaction technology have been advancing rapidly. The wide adoption of touch-enabled phones and multi-touch slates shows people's increasing affection for mouse-less interfaces, often referred to in the media as Natural User Interfaces (NUIs) [26][75]. Advances in input technology have, however, also included other types of new display hardware such as screens of many different sizes and shapes, transparent [77] or pliable [59] displays, and new types of input devices such as tangible objects, pen-based tablets, and large multi-touch surfaces. Coupled with this development a discussion on new interface design paradigms has gained attention. Discussions about post-WIMP interfaces were introduced as early as the 1990's [17][51] with the goal of making interfaces as invisible as possible - to minimize the gap between a person's intent and the execution of the intent. Examples of these post-WIMP interface paradigms include instrumental interaction [6], proxemics [28], touch-based [79], and sketch-based interaction [53].

Despite the recognition of the importance of interaction to InfoVis, comparatively little has been done to take advantage of the advances made in the interaction design community. InfoVis systems largely remain operational using the classic desktop, mouse, and

- Bongshin Lee and Nathalie Henry Riche are with Microsoft Research, EMail: \{bongshin,nath\}@microsoft.com.

- Petra Isenberg is with INRIA, E-Mail: petra.isenberg@inria.fr.

- Sheelagh Carpendale is with the University of Calgary and performed this work as a consultant for Microsoft Research, E-Mail:

sheelagh@cpsc.ucalgary.ca.

Manuscript received 31 March 2012; accepted 1 August 2012; posted online 14 October 2012; mailed on 5 October 2012.

For information on obtaining reprints of this article, please send emailto:tvcg@computer.org. keyboard setup. With this setup, principles of "point and click" WIMP GUIs (graphical user interfaces based on windows, icons, menus, and a pointer) and direct manipulation are the most common underlying models to design InfoVis interfaces. We argue that postWIMP interfaces are still relatively underexplored in InfoVis and could augment or complement WIMP for InfoVis for several reasons: a) A well-known critique of WIMP interfaces is "drowning in functionality" where options have to be found in multi-step hierarchical menus or between multitudes of buttons, checkboxes, and other widgets [17]. InfoVis often has complex sets of data-, representation-, and presentation-centric options, and thus "drowning in functionality" is a concern for the usability of InfoVis systems.

b) InfoVis often deals with multi-dimensional data. The mapping of multi-dimensional data tasks to $2 \mathrm{D}$ widgets may not be particularly natural. An example where this becomes evident is widgets for 3D navigation in information spaces [17].

c) InfoVis-even if designed for a concise overview-can quickly achieve a high-level of visual complexity which may further conflict with WIMP interfaces [17].

d) InfoVis is moving into new contexts (e.g., museums, meeting rooms, public spaces, and hand-held devices) and targeting a broader range of audiences with requirements of "anytime/anywhere" data access. It is known that WIMP interfaces are not the most appropriate interface for many display or analysis environments [75].

Given these possibilities of post-WIMP interfaces for InfoVis, an in-depth discourse and further research about alternative visualization interactions is needed. One example in this direction is a recent discussion by Elmqvist et al., which begins to advocate more careful interaction design and attempts to characterize successful fluid interaction in InfoVis [21]. Similarly, our goal in this paper is to reflect more broadly about the role of more "natural" interaction for InfoVis and to provide opportunities for future research.

We organize this paper as follows. We first summarize existing interaction models proposed by the HCI community, review how the InfoVis community classifies InfoVis-specific interactions, and describe the advances both in hardware and software technologies supporting post-WIMP interfaces. This process led us to consider missing perspectives and reflect on the role of interactions in InfoVis. From this, we derived design considerations in which we look at interactions from a novel angle, taking into account the entire spectrum of interactions that can be of value to consider in InfoVis. Finally, we discuss opportunities and challenges that guide us to future research directions. 
2

\section{HCI INTERACTION MODELS VS. INFOVIS INTERACTION} Classifications

Researchers in both the HCI and InfoVis communities have been developing a fairly large number of interaction techniques. Yet, compared to representation techniques, significantly less attention has been paid to leveraging possibilities of novel interaction modalities and models in InfoVis. In order to better understand the gap of research on novel interactions in InfoVis, it is important to note the difference on how the two research communities reacted to building a better understanding of interaction.

In order to facilitate this discussion we propose a working definition of interaction since interaction is one of those elusive terms that is extremely hard to define. Our definition is based on temporality where an interaction has a start and an end (Figure 1). The start is usually framed as an intention on the part of a person; however, some interactions such as some proxemics do not require this. The second step is usually an action performed by the person. This can range from thought or speech to any kind of movement, and can involve objects. The third step, which often overlaps extensively with the second step, involves system response in terms of functionality or reaction. The last step, which again may or may not be present, involves feedback.

Throughout the paper, we also use the definition of an interaction model by Beaudouin-Lafon [6], i.e., an interaction model is a set of principles, rules, and properties that guide the design of an interface. Such a model describes how to combine interaction techniques in a meaningful and consistent way and defines the "look and feel" of the interaction from a person's perspective.

\section{$2.1 \quad \mathrm{HCl}$ Interaction Models}

Considering the temporal spectrum of an interaction as in Figure 1, HCI interaction models tend to focus on the perspective of an interacting person. That is, the models describe and group the things that a person can do rather than the result of the action (i.e., system response or reaction).

\subsubsection{Windows, Icons, Menus, and a Pointer (WIMP)}

WIMP interfaces are descendants of the Xerox Star interaction model [63] initially started with simple principles:

- Spatial divisions are simple and either use tiling or overlapping rectangles (i.e., Windows),

- Items not in current use can be represented by small icons,

- Command controls can be replaced by menus, and

- Pointer interaction allows movement between spatially separated interface items.

WIMP interfaces have been very successful commercially and have reached a broad range of audiences for a wide range of applications by providing simple, easy-to-learn, and easy-to-use "point-and-click" interaction. They are also well supported by current GUI builders and interface toolkits, so their development can be completed relatively effortlessly according to specified standards.

\subsubsection{Direct Manipulation}

In the early eighties, Shneiderman investigated why certain interactive systems received glowing enthusiasm from people. To describe the features that promoted people's satisfactory reactions, he developed a model called direct manipulation that can be characterized by four principles [60].

- Continuous representation of objects of interest (object of interest should always be present),

- Physical actions (e.g., movement and selection with an input device that promotes physical movement such as mouse, touch screen, etc.) instead of complex syntax,

- Rapid, incremental, and reversible operations with an immediately-apparent effect on the objects of interest, and

- Incremental approach to learning that permits usage with minimal knowledge and an expansion to more complex interactions.

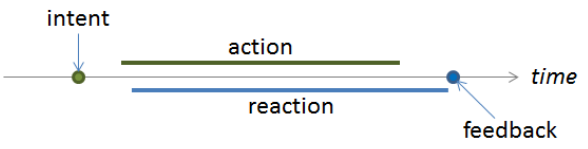

Figure 1: Temporal spectrum of an interaction.

Direct manipulation is not tethered to WIMP interfaces and therefore it has been applied to other types of interfaces including post-WIMP [17]. However, due to the dominant use of mouse and keyboard for the traditional desktop environment, direct manipulation has often been tightly coupled with WIMP interfaces. They together have dominated the interface design world and remain the most predominant interaction paradigms. For example, dynamic queries are one of the most commonly used direct manipulation interaction techniques for interactive visualization systems because they enable people to explore relational data without using complex languages such as SQL queries [76]. With dynamic queries, people can formulate queries by manipulating widgets such as check boxes and double range sliders, and immediately see the query results.

We agree that direct manipulation systems not only offer a more satisfying experience but also enable people to concentrate on their tasks [60]. However, several issues also exist. First, as Frohlich argues, direct manipulation is not abstract enough to fully express people's intentions [23][24]. Second, it does not scale for more complex applications because we cannot just add more and more directly manipulable widgets such as sliders and buttons [62]. Third, while it is more direct than the previous command-line interfaces, it is often not really a direct manipulation in that in many cases people manipulate interface elements such as menus and widgets rather than the objects of interest.

\subsubsection{Post-WIMP}

A few researchers have promoted the development of a next generation of interfaces called post-WIMP interfaces [6][7][17][51]. In 1993, Nielsen first suggested these to be non-command interfaces, which may go beyond the standard WIMP paradigm to involve elements like virtual realities, sound and speech, pen and gesture recognition, highly portable computers, etc. [51]. A few years later, van Dam defined post-WIMP as interfaces "containing at least one interaction technique not dependent on classical 2D widgets such as menus and icons" [17]. Not surprisingly, over the last two decades, a significant amount of research has been conducted to develop new interfaces that diverge from WIMP and/or direct manipulation.

\subsubsection{Post Direct Manipulation}

While there has not been a model referred to as post direct manipulation, there has been a series of efforts to expand and refine the idea of direct manipulation, attempting to overcome the issues discussed above. Here, we group these extensions and variations of direct manipulation as post direct manipulation interfaces. To encompass the wide range of emerging graphical interaction techniques, Beaudouin-Lafon introduced a new interaction model, called instrumental interaction [6], which extends and generalizes the principles of direct manipulation [60]. It is inspired by the way we use instruments to manipulate objects of interest in the physical world. Objects of interest called domain objects are manipulated with computer artifacts called interaction instruments. To help people evaluate and compare alternative designs, he also introduced three properties to evaluate these instruments [6]:

- Degree of indirection: a two-dimensional measure of the spatial and temporal distance introduced by instruments,

- Degree of integration: the ratio between the degrees of freedom of the instrument and the input device, and

- Degree of compatibility: a measure of similarity between actions on the instrument and the feedback on the object.

Klokmose and Beaudouin-Lafon further expanded the model and introduced ubiquitous instrumental interaction along with the VIGO (Views, Instruments, Governors, and Objects) architecture that 
allows multiple people to interact with multiple devices through diverse interfaces spanning multiple surfaces [44].

Jacob et al. proposed a framework, called Reality-based Interaction, which tried to unify emerging post-WIMP interaction techniques [42]. Their underlying motivation is that the new interaction techniques draw strength by building on people's knowledge of the everyday, non-digital world to a much greater extent than before. Their framework focuses specifically on the following four themes from the real world:

- Naïve Physics: people have common sense knowledge about the physical world,

- Body Awareness \& Skills: people have an awareness of their own physical bodies and possess skills for controlling and coordinating their bodies,

- Environment Awareness \& Skills: people have a sense of their surroundings and possess skills for negotiating, manipulating, and navigating within their environment, and

- Social Awareness \& Skills: people are generally aware of others in their environment and have skills for interacting with them.

Kwon et al. also believed that a coherent underlying principle has been missing although several variations (or improvements) of direct manipulation have been developed over the years [47]. They proposed a unifying model, called surrogate interaction, to address three problems - access, multiple objects, and intangible properties - in direct manipulation. The surrogate is an archetype of the domain objects, which will be immediately affected by any changes done to the surrogate. Since interaction is done through surrogate objects, this introduces a small degree of indirection between people and domain objects [47]. However, it achieves higher degree of compatibility by integrating many more interface components for controlling the attributes of the domain object in the visual representation of the surrogate. In other words, surrogate interaction tries to strike a balance between indirection and compatibility in the interface.

\subsection{Interaction Classifications for InfoVis}

Discussions and research on interaction for InfoVis have focused on interaction frameworks and taxonomies. Due to the difficulty in concretely defining interaction, they were often done in the context of data types and tasks [32][45] or the InfoVis pipeline [12][15][64]. Note how this differs in focus from the models presented in the HCI literature; the HCI models consider the person in the interaction loop while the InfoVis taxonomies consider the system reaction or the resulting functionality part of the interaction timeline (steps 3 and 4 in our definition of interaction). With this focus, InfoVis taxonomies largely discuss interaction from the viewpoint of interaction tasks to be performed with and on the data, or from interaction techniques that can be performed on the data or its visual representation.

\subsubsection{InfoVis Interaction Taxonomies}

Several researchers have published taxonomies of interaction techniques for data exploration. They are often mixed and discussed interchangeably with those related to an analyst's tasks with a visualization. For example, Shneiderman's mantra [61]: “overview first, zoom and filter, then details-on-demand" has been discussed both as abstract low-level interaction techniques (as in [81]) and as more general information analysis tasks (as in [61]). An extensive overview of interaction taxonomies from an InfoVis perspective has been compiled by $\mathrm{Yi}$ et al. (see Table 1 in [81]) and we refer the interested reader to this overview. Yi et al. also proposed their own categories of interaction techniques based on a person's data interaction intent-select, explore, reconfigure, encode, abstract/elaborate, filter, and connect. In addition, they give a few example interaction techniques in each category that have been implemented in different InfoVis systems. Note that, while Yi et al. start by thinking about the very beginning of our interaction timeline-that of personal intent-they frame that intent by what one would like to do to the data in the visualization. Here the intent is to have the system respond. The actions that might be needed to affect this response are not explicitly specified-supporting a wide range of interaction variations.

Heer and Shneiderman [32] recently proposed a taxonomy of 12 task types grouped into three higher-level categories - data \& view specifications, view manipulation, and process \& providence. Similar to Yi et al. [81], they discuss interaction techniques that can be used to support these tasks (e.g., using a lasso for a selection task).

Isenberg et al. [41] reported a number of analytic problem solving processes around visualization from an exploratory study of individuals and small groups. These differ from the ones mentioned above as they take both interactions with the visualization artefacts and with group members into account. Processes that directly involve interaction with a visualization included browse, parse, select, and operate. Those which occurred around an artifact (without necessarily direct physical contact) included discuss collaboration style, establish task strategy, clarify, and validate. This collection of processes mixes both more data-centric and people-centric interaction around data and its visualization and, thus, differs from previous taxonomies. However, many of the found processes relate to those found in the sensemaking cycle by Card et al. [12].

\subsubsection{Interaction and the InfoVis Pipeline}

In contrast to the taxonomies discussed above, a number of researchers have discussed the place of interaction throughout the process (or pipeline) of mapping data to visual form. This type of interaction is distinct from the one above as it focuses on the perspective of authoring (interactions for creating a view) while many task and technique taxonomies mentioned above focus onalthough not exclusively-an analyst's interactions when interpreting a display [13].

Card et al. [12], for example, indicate in their "reference model for visualization" (Figure 2) that interaction can be applied at four distinct stages in the process. They give examples of operations that can be applied at these four stages without going into detail on specifically implemented interaction techniques. Chi and Riedl [15], in contrast, developed an operator interaction framework in which they strove to integrate all possible visualization operations with the visualization pipeline. They classify the operators of the framework according to their state within the Card et al. pipeline (Figure 2). For example, data stage operators include those for value-filtering or subsetting. Furthermore, the authors discuss the relationship of operators to direct manipulation: the closer an operator is to the view in the pipeline, the higher the possible amount of direct manipulation.

Subsequently a variation in this pipeline was introduced by both Carpendale [13] and Spence [64], which adds on another type of operation before the view transformation step: presentation transformation. These transformations allow the viewer to change the presentation of a given representation without changing the underlying information content.

\subsection{Advances in Natural User Interface (NUI)}

No matter how simple, easy-to-learn, and easy-to-use, an interface still sits between people's intent and execution of their intent. For example, while instrumental interaction [6] extends and generalizes principles of direct manipulation to cover a broad range of graphical interaction techniques, there still exist interaction instruments between people and target objects. In other words, an interface still

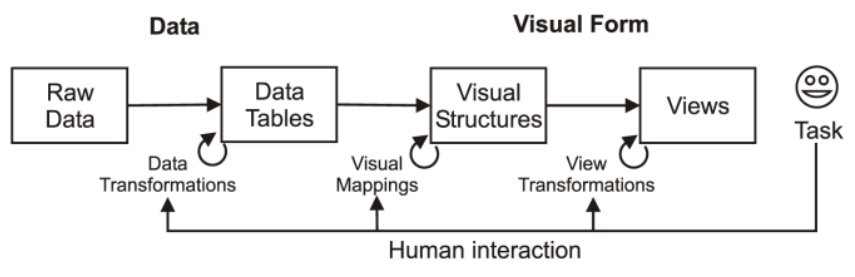

Figure 2: Card et al. pipeline [12] or their "reference model for visualization." 
imposes a layer of cognitive processing for people. Researchers have been trying to minimize the mechanics of manipulation and the cognitive distance between intent and the execution of the intent so that people can focus on the task not the interface for specifying the task. As a result, HCI researchers have been developing a wide range of post-WIMP interfaces. One of the earliest of these existed even before the WIMP interfaces era-the Sketchpad system made it possible for people to communicate with a computer through the medium of line drawings [68].

Ever since, especially over the last decade, we have observed advances both in hardware and software technologies supporting the non-mouse-based interactions that are part of post-WIMP interfaces. Furthermore, we are now facing a new paradigm called Natural User Interfaces (NUIs), which is generally used to describe those interfaces that promise to reduce the barriers to computing further [75]. There are a variety of new more "natural" (as defined through people's experience of interacting in the physical world) approaches to interaction including pen, touch, gesture, speech, and sketch. When first introduced, iPhone (and now iPad) interaction enabled an entirely new experience based on a multi-touch display and innovative software that let people control everything with their fingers. Other multi-touch devices such as multi-touch monitors and slates have become readily available.

Hinckley et al. also demonstrated that the combination of pen and touch provide not only powerful new tools but also more "natural" interaction [35]. They argue that one device cannot suit all needs and that some are inherently more natural than others for certain tasks (e.g., using a pen rather than a finger for writing). From the hardware side this notion is also now being increasingly supported. Perceptive Pixel, for example, recently released $27 " / 55 " / 82$ " capacitive displays that support both pen and touch with a capability of detecting an unlimited number of simultaneous touches [54].

Pen technology (e.g., Anoto [2]) enables researchers to explore ways to bridge the gap between paper and the digital world. For example, the NiCEBook combines the flexibility and simplicity of note-taking on paper with the benefits of a digital representation (i.e., structuring and reusing notes) [10]. The NiCE Discussion Room enables people to create, access, and share information and media from diverse sources to facilitate group discussions by integrating digital and paper tools into a cohesive pen-based system [30].

The release of the Kinect for Windows SDK [50] in June 2011 has garnered much attention not only from researchers but also from enthusiasts [26]. For example, Connolly (an independent software developer) implemented and demonstrated the image manipulations featured in Steven Spielberg's 2002 futuristic film, Minority Report. His system supports moving different images on a small bank of screens up and down, in and out, and sorted through threedimensional image arrays, using gestures alone. An increasing number of projects similarly use the Kinect technology in a wide range of domains including games, education, music, etc. with the hope to make interaction with digital artifacts natural by leveraging our familiarity with interaction in physical space.

Due to the advancement in speech recognition technology utilizing various personalization techniques (e.g., [8][25]), a growing number of applications now offer multi-modal interfaces leveraging speech. They attempt to take advantage of humans' ability to effortlessly communicate through voice. For example, Apple's Siri [3] which uses Nuance Dragon [52] allows people to ask it to do things just by talking naturally with Siri, and the virtual avatar even responds using quasi-natural language.

However, these new types of "natural" interaction seem to have bypassed the field of InfoVis, in spite of the continually increasing demand for visual access to data.

\footnotetext{
${ }^{1}$ The word "natural" is often used in the literature to assign a specific quality to novel interaction modalities (natural user interface-NUI). We acknowledge that its use is problematic as a distinguishing factor to traditional interfaces Here we use the word to describe a general interaction experience evoked on the person including instant feeling of success and ease of use.
}

\section{Interaction Design Considerations}

Our goal is to encourage special attention to the role and design of interaction in InfoVis. We propose to look at interaction from a novel and broader angle, taking into account the entire spectrum of interaction (Figure 1). We define design considerations (i.e., a set of dimensions) that not only encompass existing interaction techniques but also capture the capabilities of newly available interface and interaction technologies (i.e., what is commonly referred to as NUI or post-WIMP). These dimensions cover important aspects of both existing and newly available interaction techniques even though they are not exhaustive.

We noted above that the HCI community has created interaction models based upon the actions a person takes, while the InfoVis community has taken a more data and task-centric approach, considering the effect of a person's actions on the data and its representation and presentation. In our interaction design considerations we take a human-centric approach instead and do not specify data or tasks. We start by considering three principle dimensions - the individual, the technology, and the sociality - and then devote a discussion to the interplay between these. Each of these principle dimensions has sub-dimensions (Table 1). Important factors from the perspective of the individual are degree of intent, interaction distance, freedom of expression, and impact of system feedback. Under the technology, we have three sub-dimensions: input type, input resolution, and output type. The third set is about social aspects of interaction and includes collaboration group size, collaboration context, collaborative distance and latency, and collaboration interaction feedback. The fourth is a dimension that cuts across the first, second, and third sets, and represents the interplay between people and technology. Here, we discuss embodiment and instrumental interactions.

In this section, we present a definition of each dimension along with a brief discussion about its relevance to InfoVis.

\subsection{About the Individual}

The dimensions in this set are concerned with how a person can interact with InfoVis systems without focusing on the interactionenabling technologies.

\subsubsection{Degree of Intent}

In the past it has always been assumed that a person initiated an interaction with a particular intent — now that is not always the case. For example, with proxemic interaction [28], systems react proactively when people are getting close to the system (possibly engaging the person to interact even if it he/she did not intend to do so). Also, tangible devices can react to certain types of input-such as physical rotations - and trigger interactions that may not have been intended by the person who picked up the device.

So far, InfoVis systems almost exclusively focused on explicitintent driven interaction. Yet, with the emergence of more casual interaction such as interaction with ambient displays, interaction initiated without intent could be an effective means to get people to notice and become engaged with data displays.

\subsubsection{Interaction Distance}

This dimension captures the physical distance between the source of interaction and the target objects. Interactions may be triggered by direct contact, from across the room, from another room, or at one extreme, from another country. Body gestures and speech can invoke such remote commands, and afford interaction at a high degree of physical distance. Playing with such interactions opens up interesting directions for InfoVis, allowing data visualization sent to a remote public display through a smartphone to share, for example. Visualizations of real-time twitter streams are an example where the interaction distance is large but can be of considerable local interest.

On a much more local level, digital objects can be interacted with through a glass pane on most touch devices and offer a more direct interaction. An even more direct method of interacting with 
Table 1: Design considerations: four principle dimensions and their sub-dimensions along with examples.

\begin{tabular}{|l|l|l|}
\hline \multicolumn{1}{|c|}{ Principle Dimensions } & \multicolumn{1}{|c|}{ Sub-dimensions } & \multicolumn{1}{c|}{ Examples } \\
\hline \multirow{4}{*}{ About the Individual } & Degree of Intent & explicit vs. implicit (e.g., proxemics [28]) \\
\cline { 2 - 3 } & Interaction Distance & physical contact, remote (e.g., across the room, another country) \\
\cline { 2 - 3 } & Freedom of Expression & high (e.g., freeform sketch) low (e.g., menus, buttons) \\
\cline { 2 - 3 } & Impact of System Feedback & high bandwidth (e.g., visual) low bandwidth (e.g., sound) \\
\hline \multirow{4}{*}{ About the Technology } & Input Type & pen, touch, speech, (body) gesture \\
\cline { 2 - 3 } & Input Resolution & high (e.g., mouse) low (freeform in-air gesture) \\
\cline { 2 - 3 } $\begin{array}{l}\text { Social: Interactions between } \\
\text { People }\end{array}$ & Output Type & $\begin{array}{l}\text { mobile devices, surfaces and tabletops, wall-size displays, speakers (or } \\
\text { headsets), haptic devices }\end{array}$ \\
\hline \multirow{3}{*}{$\begin{array}{l}\text { The Interspace between a Person } \\
\text { and the Technology }\end{array}$} & Collaboration Group Size & two, three, or more \\
\cline { 2 - 3 } & Collaboration Context (Style) & one-to-many, many-to-many \\
\cline { 2 - 3 } & Collaborative Distance and Latency & co-located vs. remote \\
\cline { 2 - 3 } & Collaboration Interaction Feedback & workspace awareness, situation awareness, common ground \\
\cline { 2 - 3 } & Embodiment & finger proxies [78], sticky tools [31], touch projector [9] \\
\hline
\end{tabular}

visualizations is offered by tangible InfoVis. Here, touch requires people to physically be in contact with the target objects. Currently InfoVis systems commonly focus on an interaction distance defined by mouse, keyboard, or screen setups. However, as discussed above, the space of possibilities for interacting with different data modalities from varying distances is large. Expanding the distance of an interaction could potentially impact how well the data is understood but also how visible the data can be made to groups of people. Thus, considering interaction distance can impact more than just the data interaction experience itself and also make a visualization more accessible to people.

\subsubsection{Freedom of Expression}

Freedom of expression as a concept refers to people's ability to effortlessly express their intent through varying means, and one of the characteristics of what is often discussed as post-WIMP or NUI is the increased freedom of expression they bring to people. In contrast, WIMP interfaces offer people a very limited freedom to express different types of interactions within the system, many of which have been chosen by the interface designer and developer. Freedom of expression can be particularly important for InfoVis to consider as core components of InfoVis systems (such as search, selection, and filtering) can be often difficult to describe using commands alone. High freedom of expression can potentially support types of analyses that an interface designer has not directly provided for in a system.

\subsubsection{Impact of System Feedback}

Impact of system feedback relates to human perception bandwidth in processing the system feedback (i.e., output). For example, people can process much more visual output from high resolution screens (in parallel) than haptic or sound (serial). For this reason, InfoVis largely focused on providing visual feedback by having systems render data on a screen. However, the combination with other types of feedback is worth considering. A small community of researchers is actively pursuing research on how data can be provided through haptics [46] or sound [82]. This can be beneficial for people who cannot rely on visual output or for augmenting visual output to provide one or more additional dimensions for displaying data. Similarly, the exploration of how one can in return interact through haptics or sound with data is worth considering. As such, while "visualization" is often synonymous with visual rendering, interaction between a person and data through other means may be viable additions or alternatives.

\subsection{About the Technology}

The dimensions in this set consider the technology that enables interactions. The components of these dimensions are constantly in flux due to the frequent introduction of new technology.

\subsubsection{Input Type}

The input type dimension covers a wide range of input types (or modalities) available. These include pen, touch, speech, (body) gesture, tangible, etc. Of these novel input devices, InfoVis systems have taken only little advantage. Most common are probably systems that utilize touch interactions [20][22][58][71]. Only a few systems utilize speech (mostly natural language) [16][67] and we are starting to see a few efforts utilizing pen [11][14]. Understanding the benefits of different technologies is not only important as a means of system input; novel technology can also be used and reappropriated to aid in the evaluation of InfoVis systems. For example, brain-computer interfaces have been recently used in this context [1].

\subsubsection{Input Resolution}

Input resolution concerns how reliably interactions can be recognized, i.e., the degree of accuracy a person can achieve in terms of the precision of his/her interactions. Each input type has different affordances that impact its resolution. For example, mouse interaction is highly reliable and supports high-precision. A pen can provide precise interactions and be comparable to the mouse in this regard. In contrast, freeform gestures are in general difficult to recognize reliably due to the lack of uniformity in their invocation. For example, everyone may move his/her body in a somewhat different way to specify a same in-air gesture. Touch reliability is currently somewhere in between that of a mouse and that of a free form gesture (but close to a mouse).

InfoVis systems so far have been mainly designed for mouse interaction, which provides a very high precision along with hover capability. Yet, current touch systems are becoming more reliable and finger touches are increasingly consistently recognized. However, issues around the 'fat-finger' problem still remain in the selection of smaller objects, which are common in data representations. As small screen multi-touch displays such as smartphones and slate devices become more and more popular, it is important for InfoVis to consider interactions that can be reliably recognized through low precision input.

\subsubsection{Output Type}

The output type dimension attempts to cover different types of output devices where we consume the output from InfoVis systems. This includes small and large screens (e.g., mobile devices and wallsize displays), surfaces and tabletops, speakers (or headsets), haptic devices, etc. Given InfoVis' focus on visual output, other than regular monitor screens, the community has mostly investigated the use of large screens or tabletops to represent data. Different screens offer different types of opportunities (e.g., people carry smartphones everywhere nowadays) and their consideration can be particularly valuable to pursue in InfoVis as the requirements for anytime/anywhere data access increase. 


\subsection{Social: Interactions between People}

The dimensions in this set are concerned with how a group of people collaboratively interact not only with InfoVis systems but also with each other within the group.

\subsubsection{Collaboration Group Size}

Assuming a possibility for all people to synchronously interact, several challenges arise in regards to group size. One is the problem of awareness discussed later in Section 3.3.4. Another is more technical in nature. When concurrent interaction is possible, data structures and visual representations need to be designed such that they can be modified and accessed at the same time. Algorithms for data processing and rendering need to be adapted so that they still allow for real-time interaction even as group size grows and more interaction operations are received concurrently. This may require the development of dedicated visualization libraries as done for the Lark system [71]. Finally, research on cognition has shown that groups need to employ various coordination mechanisms when transmitting information and that information representations change as they are passed from one group member to the next [38]. In this vein Liu et al. argue that the "standard one-person-one-computer InfoVis model is over-simplified," that the larger cognitive system needs to be considered, and that interaction and coordination are important research topics for social visualization [48].

\subsubsection{Collaboration Context (Style)}

Collaborative work comes in many different contexts. For example, the situation can be one-to-many or many-to-many. Even a one-tomany situation can vary between scenarios where one person is communicating with several people to where one person is keeping track of several people who are working in parallel.

The context dimension attempts to capture the social context of interaction. For example - while mobile or slate devices are likely to be used by an individual-tabletops, surfaces, or large wall displays could be used for multiple people. In fact, previous research shows that the types of gestures people use on interactive surfaces depend on social context [36]. Factors such as the need to reach different parts of a screen or the possibility of accidentally making physical contact with others affects how an interface should be designed and which interaction mechanisms are most appropriate for a given context. In addition, the feedback and feedthrough of interactions is impacted by context. For example, speech interaction may not be desirable in a public space or in a work setting where it could create privacy concerns or be disturbing for others.

\subsubsection{Collaborative Distance and Latency}

There has been considerable discussion about the different challenges of co-located and distributed collaboration [56]. Furthermore, both of these also vary with whether they are synchronous or asynchronous. Interaction challenges exist for each time and space dimension: they have to be synchronized and integrated in various ways and also be potentially enhanced with awareness and common ground features as briefly discussed next. Visualization toolkits currently do not offer much support for designing interactions across these different collaborative settings.

\subsubsection{Collaboration Interaction Feedback}

This concept has been most frequently discussed as awareness [29]. Feedback in a collaborative setting must cover not only feedback about the data and information-centric results but also about the ongoing actions of the collaborators. For example, with only two people collaborating, it is relatively simple to keep track of whose actions are having which effect. As the number of people in a group goes to three and above this gets more challenging. This means that it may no longer be enough to simply design for the possibility of synchronous interaction but also that awareness tools will have to augment the interaction [39]. For co-located settings this problem is particularly acute for InfoVis systems. In contrast to other types of scenarios, InfoVis tasks often require close scrutiny of large information spaces and, thus, it becomes even more difficult to remain aware of others' actions in a space-even though there may be a joint workspace between them. Previous research has also shown that both different types of groups and different group members can have vastly different approaches to data analysis [40]. Thus, providing high awareness is particularly important for collaborative InfoVis interfaces. Within an InfoVis system, addition of extra 'collaboration-level' visualizations needs to be considered carefully in order to avoid interfering with the data visualization.

\subsection{The Interspace between a Person and the Technology}

The dimensions in this set build a bridge between the first three sets and discuss the space between them.

\subsubsection{Embodiment}

The embodiment dimension refers to the degree to which a person feels that the technology is an extension (or part) of them. For example, car racers know the size and shape of their car (i.e., they know to the centimeter if they can make it through a gap), and thus can take what seems like an incredible risk. Gamers have fully mastered the use of console controllers and do not have to look at them while playing. Embodied interaction as such makes use of a number of human capabilities including motor memory, peripheral vision, optical flow, focal attention, and spatial memory [19]. Ball and North [4] argue that these capabilities are particularly applicable when data is displayed on very large displays and advocate physical navigation as a form of embodied interaction with data. Similarly, other types of embodied interactions could be investigated that make interaction with data particularly effective and "natural."

\subsubsection{Instruments}

This dimension captures the use of instruments (or tools) such as a pen or other physical objects. For example, some interactions require gloves or sensors which may need to be attached to a body, whereas touch interaction does not require additional objects. These types of instruments often augment human capabilities by providing more controls. For example, as mentioned before, use of a pen enables a higher input resolution than touch (by fingers) input.

Tangible objects are often designed to look like corresponding objects in the real world and have very specific affordances. These convey the possible ways of interaction and help people learn how to interact with an object. For example, a digital pen for interactive whiteboards looks like a regular marker and people immediately know how to write with it. However, tangible interactions are one of the least explored areas in InfoVis. As InfoVis deals with abstract data, which does not necessarily have a spatial representation that corresponds to something in the physical world-it may be a particular challenge to find intuitive and natural instruments to interact with data.

\section{Opportunities and Challenges}

In this section, we describe opportunities and a call for increasing research attention on novel interaction modalities, drawn from the reflection on the design considerations described above, and grounded in examples of existing interaction techniques. It is important to note that our intention is not to argue that post-WIMP is better than WIMP, which has its own place. We instead focus on how post-WIMP and more "natural" interaction paradigms could augment (or complement) traditional WIMP interfaces for InfoVis.

\subsection{Go Beyond Mouse and Keyboard}

As previously mentioned, current InfoVis systems are still largely focused on the use of mouse and keyboard for interacting with data. They also often rely on numerous menus and complex control panels. Exploring different input modalities may allow the use of more degrees-of-freedom interaction and thus reduce the number of 
necessary UI components. This can help analysts focus their attention to their main task, visual exploration of the data rather than the manipulation of an interface.

Gestural interaction, a characterizing component of post-WIMP or NUI interfaces, is one example of an interaction technique that researchers have explored for InfoVis benefits. Frisch et al. [22] researched the suitability of pen and touch gestures and bimanual interaction for editing node-link diagrams on tabletops. Their goal was to work towards a gesture set that would make diagram manipulation both "more efficient and effective." Dwyer et al. [20] demonstrated that multi-touch interactions to manipulate node location could encourage people to "think with their hands." Schmidt et al. [58] broadened graph interaction possibilities by using multiple contact points for better graph edge detangling and collaboration.

While there have been a handful of sketch-based interactions (e.g., [37][57][74]) relevant to InfoVis, just a few of them utilized interaction modalities other than the mouse to draw lines. NapkinVis uses pen gestures to support free-flowing, fast, and effortless visualization construction [14]. SketchVis (Figure 3a) leverages hand-drawn sketch input to quickly explore data in simple charts without the use of menus or widgets [11].

Also, there have been only a few attempts to incorporate natural language and speech-based interfaces in InfoVis systems even though multi-modal interfaces are heavily researched in the HCI community. Cox et al. use natural language as input to an InfoVis system to support query formulation without menus or knowledge of SQL syntax [16]. Articulate, another InfoVis system with a speech component, allows people to verbally describe and manipulate what they want to see [67]. Using speech for data analysis has two main potential benefits. First, it allows people to interact with objects without direct contact. Secondly, they can rely on a vocabulary that already carries meaning and may not have to be learned. This modality, thus, has a lot of promise for the InfoVis community.

Even though new input modalities, such as the few examples described above, in general have great potential to lead to more "natural" interaction for InfoVis, little is known on how people would use and want to combine them to explore data or create visualizations. Furthermore, we have very little understanding on the most important benefits and issues of new input modalities. To successfully leverage novel modalities and devices for InfoVis, we need to gain a better understanding of their strengths and weaknesses. Furthermore, combining multiple modalities may yield new helpful interactions because different modalities can complement each other [35]. Hence, one promising research avenue for InfoVis is to investigate how to combine different modalities.

\subsection{Provide High Freedom of Expression}

Freedom of expression of interaction intent is an extremely important component for more "natural" interaction and relates highly to InfoVis systems. For example, formulating queries, one of the most important, commonly used but difficult tasks in InfoVis, has been highly relying on WIMP-based dynamic queries as discussed in Section 2.1.2 [76]. By providing higher freedom of expression through post-WIMP interfaces or NUIs, researchers may better support analysts in forming hypotheses and expressing questions raised during the data exploration. The systems utilizing natural language from the previous section provide examples.

There are, however, also a few other exceptions beyond speech input. QuerySketch [74] and QueryLines [57] provide high freedom of expression by allowing people to perform queries of time-series data by drawing a freeform graph as a target pattern that would be very difficult to specify mathematically or using traditional widgets and menus. Holz and Feiner's relaxed selection techniques for timeseries, designed for pen or touch input, has similar advantages. A query can be created with a single-gesture interaction [37]. A user study showed clear benefits in terms of interaction speed as well as precision and recall for their selection technique.
Facet-Streams (Figure 3b) offer high freedom of expression through a combination of touch and tangibles by making the filter/flow metaphor of Boolean queries physical [43]. People are not exposed to complex formal notations but can still harness the expressive power of facetted queries.

In summary, many alternative inputs offer multiple degree-offreedom interaction that can be leveraged to provide more freedom of expression. For example, tangible devices can be manipulated physically in 3D space offering multitudes of input dimensions and giving people an alternative and often more expressive means of defining their intent. Given that InfoVis often focuses on multidimensional datasets considering the combination of inputs with different degrees of freedom could be viable to support higher freedom of expression. In general, the possibility of freeform interaction to express intentions is one of the benefits of more natural interactions because some intensions are difficult to specify using mouse and keyboard. In addition, interaction techniques portraying a real world metaphor could be easier to learn and use. For example, it is easy to remember to draw a shape to specify a chart type (as illustrated in SketchVis [11]) and to drag links with one or more fingers to collapse them (as shown in [58]).

One thing to note is that there could be a tension between the freedom of expression and the input resolution. Currently, many novel forms of input still suffer from lower input reliability so that the recognition of gestures, for example, may not always be $100 \%$ accurate. Therefore, there are cases where providing more freedom, e.g., with freeform gestures, leads to less accurate input. It would be best if we could provide a high level of freedom while achieving a high level of recognition reliability. We should strive for striking a balance between these two dimensions.

\subsection{Take Into Account Social Aspects}

Interaction with data representations is impacted differently for groups or teams working together than for individuals. The classic desktop setup equipped with a mouse, keyboard, and screen is designed for single person interaction. In a "natural" computing environment, groups of people can collaboratively interact with the system and with each other, and easily drop in and out of a collaborative setting. Designing interaction for social data analysis settings that are constantly in flux will require more research on how to re-design interfaces and their interactions. For example, while designed to be feature-rich, the hybrid graph/matrix visualization NodeTrix [34] originally included minimal means for gestural and postural pen interaction. When the interface was re-designed for synchronous collaborative use (CoCoNutTrix [39], Figure 3c), almost all WIMP features of NodeTrix had to be removed to support for more fluid and natural interaction in groups. Interaction was provided through multiple mice and all (remaining) analysis features were made accessible such that the possibility of interference between team members could be minimized. A study confirmed that these design decisions, which moved away from the original WIMP interface, were indeed beneficial for the collaborative setting.

Thus, to design InfoVis interactions for groups it is important to take into account social aspects of how people interact around data displays. Also, there is another interplay between InfoVis and group work in general: as described in Section 3.3.4, as the group size gets bigger, support for extra interaction challenges brought up by collaboration including awareness and software infrastructure need to be tackled [40]. While there has been a recent line of work to design InfoVis systems supporting co-located collaborations [33][38][71], there are also many other opportunities to support team members with novel interaction devices. Much more remains to be done to consider a broader range of social aspects, asynchronous collaboration, as well as work and casual contexts, such as visualization use in public spaces. 


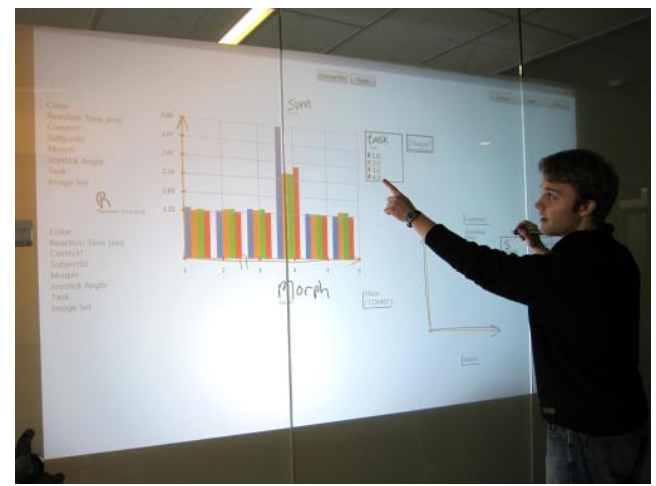

(a) SketchVis [11]

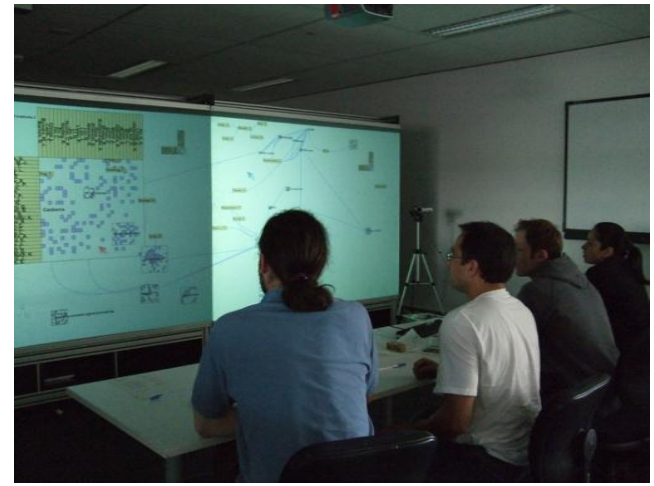

(c) CoCoNutTrix [39]

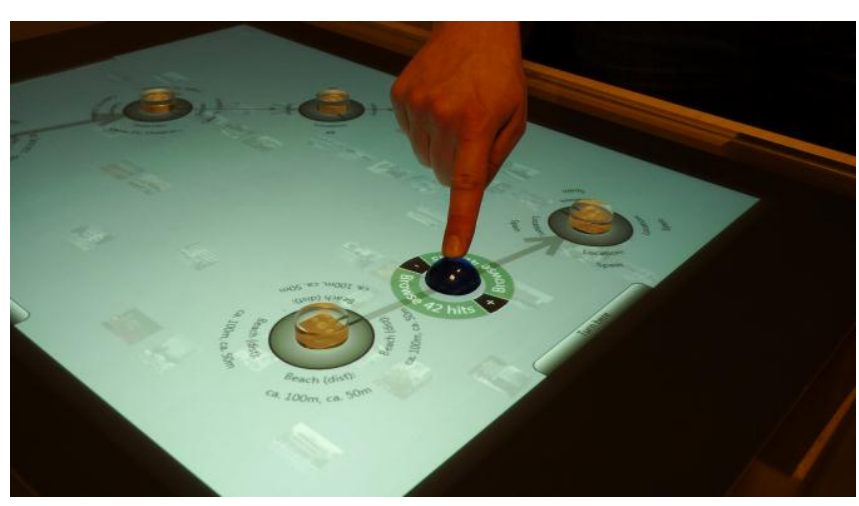

(b) Facet-Streams [43]

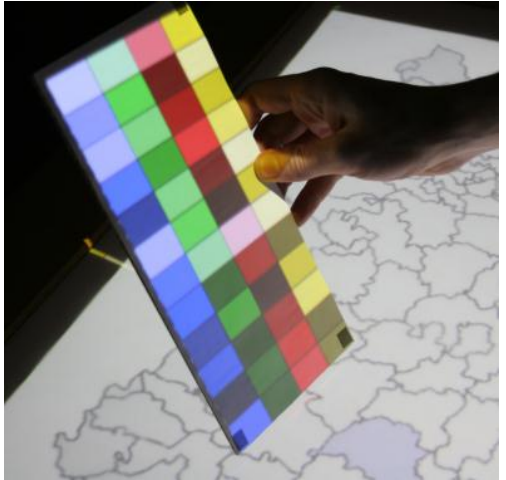

(d) Tangible Views [66]

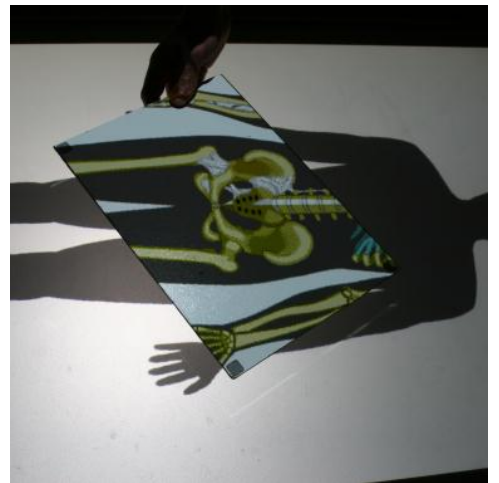

(e) PaperLens [65]

Figure 3: Example InfoVis systems leveraging "natural" interactions. These figures are courtesy and copyright of: (a) Bongshin Lee; (b) HansChristian Jetter; (c) Anastasia Bezerianos; and (d)/(e) Martin Spindler.

\subsection{Reduce the Gap between a Person and the Technology}

No matter how simple and easy-to-use an interface is, there is always a gap (i.e., indirection) between a person and the technology. By reducing this gap, InfoVis may reach a wider audience. Examples include recent research to create visualizations for the general public, supporting visual exploration and presentation of their data [5][73].

One way to reduce this gap is to use tangible objects. However, tangible interactions specifically for InfoVis are still relatively rare. Two noteworthy examples are tangible views (Figure 3d) [66] and PaperLens (Figure 3e) [65], which provide means for physical lens interaction with information spaces. The interactions are mediated through a vocabulary of physical movement of cardboard lenses. The interface dispenses almost completely with traditional menus and widgets, and is thus able to dedicate all display space to the visualization itself. Swindells et al. [69] evaluated mouse, slider, and pen devices for their ability to provide eyes-free parameter manipulation with a visualization component. The results showed that physical sliders reduced fixation time on the interface elements which seemed to free cognitive load for the main task.

One of the things new technologies offer is a way to augment human capabilities and give a person more control through the reduction of the gap between the technology and the person using it. Reducing this gap can also help enhance personal experience by going beyond physical reality (and its limitations). For example, a person can feel that the technology is a part (or extension) of him/her through embodiment and hence have better control. InfoVis systems can provide a person with instruments, through which he/she can gain the type of control needed. Also more physical interaction using one's whole body might actually help in the goal of enhancing cognitive abilities. It would be very interesting to explore further how novel means of input can enhance the data analysis experience by utilizing tangible objects or leveraging people's kinesthetic memory of data.

\subsection{Gain a Better Understanding of People's Behaviors}

The InfoVis community has been following a task-based interface design approach - focusing more on the tasks rather than people who would use the system. This approach has worked relatively well for designing systems with WIMP interfaces, which have a fixed, limited set of ways of interaction. However, with the possibility of post-WIMP interfaces supporting more freeform interactions, there are numerous additional possibilities of interaction and little is known about how people would interact with a system equipped with these new technologies. To address this issue, researchers have started to observe people's behaviors in certain situations. For example, how people naturally interact around a tabletop display with documents and search results during an investigative analysis [40], how novices would construct information visualizations when unhampered by the normal constraints of an interface [27], and how people would use pen and touch gestures for editing node-link diagrams [22]. It would be useful to make further efforts to expand our understanding of how people behave when using new technologies during data analysis and how they leverage potentially increased freedom of expression to follow their interaction intents. Better understanding of the behaviors and reactions of target audiences before or during the design of InfoVis systems may enable us to create better interaction experiences.

\section{Conclusion}

Despite the continually increasing demand for visual access to data and rapid advances in interaction technologies, InfoVis has not taken full advantage of the possibilities of evolving interaction technologies. If anything, this is particularly so for those that are generally considered to be able to provide more natural means of interaction. In this paper, we reflected upon the role of interaction in InfoVis and discuss design considerations that we hope will trigger future research. More specifically, we first discuss and relate general $\mathrm{HCI}$ interaction models to InfoVis specific interaction classifications 
based on the temporality of interactions, and discuss why the past focus of the InfoVis community may have led new technologies to be generally overlooked as new research opportunities. We looked at interactions from a novel angle, taking into account the entire spectrum of interactions. Based on this discussion to help identify underexplored areas in InfoVis interaction, we offer interaction design considerations that consider this interaction space from the perspectives of: the individual; the technology; the social aspects; and the interplay between a person and the technology. Finally, we reflected on these design considerations and described five opportunities and challenges for the InfoVis community:

- Go beyond mouse and keyboard: Since InfoVis interfaces often require a plethora of menus and controls, exploring different input modalities may make it possible to limit the number of these components, allowing analysts to focus their attention on exploring the visualization rather than operating an interface.

- Provide high freedom of expression: Current InfoVis research focuses on creating effective visual representations. While this is essential, a perhaps underexplored aspect is how to support analysts in expressing their questions and hypothesis in a fluid, highly configurable way. We might explore how to use higher freedom of expression to better support analysts in specifying hypotheses and expressing questions in an interface that would otherwise be either impossible or very difficult given only mouse and keyboard input.

- $\quad$ Take into account social aspects: This factor has already made some impact on InfoVis research as more attention is being paid to creating visualization environments that support collaborative information tasks. While it is known how important team work is to complex information tasks, there are still multiple opportunities for further research on how to best support team members with novel interaction devices.

- $\quad$ Reduce the gap between a person and the technology: This may help us reach a wider audience and offer them more power to explore data visually. This also aligns well with recent research that is exploring creating visualizations for the general public.

- Gain a better understanding of people's behaviors: The InfoVis community has been mainly discussing how to evaluate InfoVis systems after they are developed. Understanding the behaviors of target audience before or during the design of InfoVis systems may help us create better interaction experience.

In summary, these new technologies bring with them a wealth of new interaction opportunities and research directions. By leveraging advances in new interface technologies and pursuing these research questions they open up, InfoVis systems can potentially be more effective, more engaging, and thus, more widely used.

\section{References}

[1] E.W. Anderson, K.C. Potter, L.E. Matzen, J.F. Shepherd, G.A. Preston, and C.T. Silva, A User Study of Visualization Effectiveness Using EEG and Cognitive Load, Computer Graphics Forum (Proc. EuroVis), vol. 30, no. 3, pp. 791-800, 2011.

[2] Anoto - Start, http://www.anoto.com.

[3] Apple - iPhone 4S - Ask Siri to help you get things done, http://www.apple.com/iphone/features/siri.html.

[4] R. Ball and C. North, Realizing Embodied Interaction for Visual Analytics Through Large Displays, Computers \& Graphics, vol. 31, no. 3, pp. 380-400, 2007.

[5] D. Baur, F. Seiffert, M. Sedlmair, and S. Boring, The Streams of Our Lives: Visualizing Listening Histories in Context, IEEE TVCG (Proc. InfoVis), vol. 16, no. 6, pp. 1119-1128, 2010.

[6] M. Beaudouin-Lafon, Instrumental Interaction: An Interaction Model for Designing Post-WIMP User Interfaces, Proc. CHI, pp. 446-453, 2000.

[7] M. Beaudouin-Lafon, Designing Interaction, Not Interfaces, Proc. AVI, pp. 15-22, 2004.

[8] J. Bellegarda, Statistical Language Model Adaptation: Review and Perspectives, Speech Communication, vol. 42, pp. 93-108, 2004.
[9] S. Boring, D. Baur, A. Butz, S. Gustafson, and P. Baudisch, Touch Projector: Mobile Interaction Through Video, Proc. CHI, pp. $2287-$ 2296, 2010.

[10] P. Brandl, C. Richter, and M. Haller, NiCEBook - Supporting Natural Note Taking, Proc. CHI, pp. 599-608, 2010.

[11] J. Browne, B. Lee, S. Carpendale, N. Riche, and T. Sherwood, Data Analysis on Interactive Whiteboards through Sketch-based Interaction, Proc. ITS, pp. 154-157, 2011.

[12] S. Card, J. Mackinlay, and B. Shneiderman, Readings in Information Visualization: Using Vision to Think, Morgan Kaufmann, 1999.

[13] S. Carpendale, A Framework for Elastic Presentation Space, PhD Dissertation, Dept. of Computer Science, Simon Fraser University, Vancouver, Canada, 1999

[14] W.O. Chao, T. Munzner, and M. van de Panne, Poster: Rapid PenCentric Authoring of Improvisational Visualizations with NapkinVis. Posters Compendium InfoVis, 2010.

[15] E.H. Chi and J. Riedl, An Operator Interaction Framework for Visualization Systems, Proc. InfoVis, pp. 63-70, 1998.

[16] K. Cox, R.E. Grinter, S.L. Hibino, L.J. Jagadeesan, and D. Mantilla, A Multi-Modal Natural Language Interface to an Information Visualization Environment, J. Speech Technology, vol. 4, pp. 297-314, 2001.

[17] A. van Dam. Post-WIMP user interfaces, $C A C M$, vol. 40, no. 2, pp. $63-$ 67, 1997.

[18] Data Science Revealed: A Data-Driven Glimpse into the Burgeoning New Field, http://www.emc.com/collateral/about/news/emc-datascience-study-wp.pdf.

[19] P. Dourish, Where The Action Is: The Foundations of Embodied Interaction, MIT Press, 2001.

[20] T. Dwyer, B. Lee, D. Fisher, K. Inkpen, P. Isenberg, G. Robertson, and C. North, Understanding Multi-touch Manipulation for Surface Computing, IEEE TVCG (Proc. InfoVis), vol. 25, no. 19, pp. 961-968, 2009.

[21] N. Elmqvist, A. Vande Moere, H.-C. Jetter, D. Cernea, H. Reiterer, and T.J. Jankun-Kelly, Fluid Interaction for Information Visualization, Information Visualization, vol. 10, pp. 327-340, 2011.

[22] M. Frisch, J. Heydekorn, and R. Dachselt. Investigating Multi-Touch and Pen Gestures for Diagram Editing on Interactive Surfaces, Proc. ITS, pp. 149-156, 2009.

[23] D. Frohlich, Direct Manipulation and Other Lessons. In M. Helander, T. K. Landauer, and P. V. Prabhu, editors, Handbook of Human-Computer Interaction, pp. 463-488, 1997.

[24] D.M. Frohlich, The History and Future of Direct Manipulation, Behaviour \& Information Technology, vol. 12, no. 6, pp. 315-329, 1993.

[25] M.J.F. Gales, Maximum Likelihood Linear Transformations for HMMBased Speech Recognition, Computer Speech \& Language, vol. 12, pp. 75-98, 1998.

[26] G. Goth, Brave NUI world, CACM, vol. 54, no. 12, pp. 14-16, 2011.

[27] L. Grammel, M. Tory, and M.A. Storey, How Information Visualization Novices Construct Visualizations, IEEE TVCG (Proc. InfoVis), vol. 16, no. 6, pp. 943-952, 2010.

[28] S. Greenberg, N. Marquardt, T. Ballendat, R. Diaz-Marino, and M. Wang. Proxemic Interactions: The New Ubicomp? ACM Interactions, vol. 18, no. 1, pp. 42-50, 2011.

[29] C. Gutwin and S. Greenberg, A Descriptive Framework of Workspace Awareness for Real-Time Groupware, CSCW, vol. 11, no. 3-4, pp. 411446, 2002.

[30] M. Haller, J. Leitner, T. Seifried, J. Wallace, S. Scott, C. Richter, P. Brandl, A. Gokcezade and S. Hunter, The NiCE Discussion Room: Integrating Paper and Digital Media to Support Co-Located Group Meetings, Proc. CHI, pp. 609-618, 2010.

[31] M. Hancock, T. ten Cate, and S. Carpendale, Sticky Tools: Full 6DOF Force-Based Interaction for Multi-Touch Tables, Proc. ITS, pp. 145$152,2009$.

[32] J. Heer and B. Shneiderman, Interactive Dynamics for Visual Analysis, ACM Queue, vol. 10, no. 2, pp. 30-55, 2012.

[33] M. Heilig, S. Huber, M. Demarmels, and H. Reiterer, ScatterTouch: A Multi Touch Rubber Sheet Scatter Plot Visualization for Co-located Data Exploration, Proc. ITS, pp. 264-264, 2010. 
[34] N. Henry, J.-D. Fekete, and M.J. McGuffin; NodeTrix: a Hybrid Visualization of Social Networks, IEEE TVCG (Proc. InfoVis), vol. 13, no. 6, pp. 1302-1309, 2007.

[35] K. Hinckley, K. Yatani, M. Pahud, N. Coddington, J. Rodenhouse, A. Wilson, H. Benko, and B. Buxton, Pen + Touch $=$ New Tools, Proc. UIST, pp. 27-36, 2010.

[36] U. Hinrichs and S. Carpendale, Gestures in the Wild: Studying MultiTouch Gesture Sequences on Interactive Tabletop Exhibits, Proc. CHI, pp. 3023-3032, 2011.

[37] C. Holz and S. Feiner: Relaxed Selection Techniques for Querying Time-Series Graphs, Proc. UIST, pp. 213-222, 2009.

[38] P. Isenberg and S. Carpendale, Interactive Tree Comparison for Colocated Collaborative Information Visualization, IEEE TVCG (Proc. InfoVis), vol. 13, no. 6, pp. 1232-1239, 2007.

[39] P. Isenberg, A. Bezerianos, N. Henry, S. Carpendale, and J.-D. Fekete. CoCoNutTrix: Collaborative Retrofitting for Information Visualization, $C G \& A$, vol. 29 , no. 5 , pp. 44-57, 2009.

[40] P. Isenberg, D. Fisher, S.A. Paul, M.R. Morris, K. Inkpen, and M. Czerwinski, Collaborative Visual Analytics Around a Tabletop Display, IEEE TVCG, vol. 18, no. 5, pp. 689-702, 2012.

[41] P. Isenberg, A. Tang, and S. Carpendale, An Exploratory Study of Visual Information Analysis, Proc. CHI, pp. 1217-1226, 2008.

[42] R.J.K. Jacob, A. Girouard, L.M. Hirshfield, M.S. Horn, O. Shaer, E.T. Solovey, and J. Zigelbaum, Reality-Based Interaction: A Framework for Post-WIMP Interfaces, Proc. CHI, pp. 201-210, 2008

[43] H.-C. Jetter, J. Gerken, M. Zöllner, H. Reiterer, and N. Milic-Frayling, Materializing the Query with Facet-Streams - A Hybrid Surface for Collaborative Search on Tabletops, Proc. CHI, pp. 3013-3022, 2011.

[44] C.N. Klokmose and M. Beaudouin-Lafon, VIGO: Instrumental Interaction in Multi-Surface Environments, Proc. CHI, pp. 869-878, 2009.

[45] R. Kosara, H. Hauser, and D.L. Gresh, An Interaction View on Information Visualization, Proc. Eurographics-State of the Art Reports, pp. 123-137, 2003.

[46] A. Kretz, R. Huber, and M. Fjeld, Force Feedback Slider (FFS): Interactive Device for Learning System Dynamics, Proc. ICATL, pp. 457-458, 2005.

[47] B. Kwon, W. Javed, N. Elmqvist, and J.-S. Yi, Direct Manipulation Through Surrogate Objects, Proc. CHI, pp. 627-636, 2011.

[48] Z. Liu, N.J. Nersessian, and J.T. Stasko, Distributed Cognition as a Theoretical Framework for Information Visualization, IEEE TVCG (Proc. InfoVis), vol. 14, no. 6, pp. 1173-1180, 2008.

[49] Many Eyes, http://www.many-eyes.com.

[50] Microsoft Kinect for Windows | Develop for the Kinect | Kinect for Windows, http://www.microsoft.com/en-us/kinectforwindows.

[51] J. Nielsen, Noncommand User Interfaces, $C A C M$, vol. 36, no. 4, pp. 8399, 1993.

[52] Nuance - Dragon Dictation: iPhone - Dragon Dictation for iPadTM, iPhoneTM and iPod touchTM is an easy-to-use voice recognition application, http://www.nuance.com/for-business/by-product/dragondictation-iphone/index.htm.

[53] L. Olsen, F.F. Samavati, M.C. Sousa, and J.A. Jorge, Sketch-Based Modeling: A Survey, Computers \& Graphics, vol. 33, pp. 85-103, 2009.

[54] Perceptive Pixel, http://www.perceptivepixel.com.

[55] W.A. Pike, J.T. Stasko, R. Chang, and T.A. O'Connell, The Science of Interaction, Information Visualization, vol. 8, no. 4, pp. 263-274, 2009.

[56] N.H. Riche, K. Inkpen, J.T. Stasko, T. Gross, and M. Czerwinski, Supporting asynchronous collaboration in visual analytics systems, Proc. AVI, pp. 809-811, 2012.

[57] K. Ryall, N. Lesh, T. Lanning, D. Leigh, H. Miyashita, and S. Makino, QueryLines: Approximate Query for Visual Browsing, Ext. Abst. CHI, pp. 1765-1768, 2005.

[58] S. Schmidt, M. Nacenta, R. Dachselt, and S. Carpendale, A Set of Multitouch Graph Interaction Techniques, Proc. ITS, pp. 113-116, 2011.

[59] C. Schwesig, I. Poupyrev, and E. Mori, Gummi: A Bendable Computer, Proc. CHI, pp. 263-270, 2004.

[60] B. Shneiderman, Direct Manipulation: A Step Beyond Programming Languages, IEEE Computer, vol. 16, no. 8, pp. 57-69, 1983.
[61] B. Shneiderman, The Eyes Have It: A Task By Data Type Taxonomy for Information Visualizations, Proc. VL, pp. 226-242, 1996.

[62] B. Shneiderman and P. Maes, Direct Manipulation vs. Interface Agents, Interactions, vol. 4, no. 6, pp. 42-61, 1997.

[63] D.C. Smith, C. Irby, R. Kimball, and E. Harslem, The Star User Interface: An Overview, Proc. AFIPS, pp. 515-528, 1982.

[64] R. Spence, Information Visualization: Design for Interaction, Pearson, $2^{\text {nd }}$ ed., 2007.

[65] M. Spindler, S. Stellmach, and R. Dachselt, PaperLens: Advanced Magic Lens Interaction Above the Tabletop, Proc. ITS, pp. 69-76, 2009.

[66] M. Spindler, C. Tominski, H. Schumann, and R. Dachselt, Tangible Views for Information Visualization, Proc. ITS, pp. 157-166. 2010.

[67] Y. Sun, J. Leigh, A. Johnson, and S. Lee, Articulate: A SemiAutomated Model for Translating Natural Language Queries into Meaningful Visualizations, Proc. SG, LNCS, vol. 6133, pp. 184-195, 2010.

[68] I.E. Sutherland, Sketchpad A Man-Machine Graphical Communication System, Proc. AFIPS Spring Joint Comp. Conf, pp. 329-346, 1963.

[69] C. Swindells, K.E. MacLean, K.S. Booth, and M.J. Meitner, Exploring Affective Design for Physical Controls, Proc. CHI, pp. 933-942, 2007.

[70] J.J. Thomas and K.A. Cook, Illuminating the Path, IEEE, 2005.

[71] M. Tobiasz, P. Isenberg, and S. Carpendale, Lark: Coordinating Colocated Collaboration with Information Visualization, IEEE TVCG (Proc. InfoVis), vol. 15, no. 6, pp. 1065-1072, 2009.

[72] F.B. Viégas, M. Wattenberg, M. McKeon, F. van Ham, and J. Kriss, Harry Potter and the Meat-Filled Freezer: A Case Study of Spontaneous Usage of Visualization Tools, Proc. HICSS, 2008

[73] M. Wattenberg, Baby Names, Visualization, and Social Data Analysis, Proc. InfoVis, pp. 1-7, 2005.

[74] M. Wattenberg, Sketching a Graph to Query a Time-Series Database, Ext. Abs. CHI, pp. 381-382, 2001.

[75] D. Wigdor and D. Wixon, Brave NUI World: Designing Natural User Interfaces for Touch and Gesture, Morgan Kaufmann, 2011.

[76] C. Williamson and B. Shneiderman, The Dynamic HomeFinder: Evaluating Dynamic Queries in a Realestate Information Exploration System, Proc. Research and Development in Information Retrieval, pp. 338-346, 1992

[77] A.D. Wilson, TouchLight: An Imaging Touch Screen and Display for Gesture-Based Interaction, Proc. ICMI, pp. 69-76, 2004.

[78] A.D. Wilson, S. Izadi, O. Hilliges, A. Garcia-Mendoza, and D. Kirk, Bringing Physics to the Surface, Proc. UIST, pp. 67-76, 2008.

[79] J.O. Wobbrock, M.R. Morris, and A.D. Wilson, User-Defined Gestures for Surface Computing, Proc. CHI, pp. 1083-1092, 2009.

[80] Wordle - Beautiful Word Clouds, http://www.wordle.net.

[81] J.S. Yi, Y.A. Kang, J.T. Stasko, and J.A. Jacko, Toward a Deeper Understanding of the Role of Interaction in Information Visualization, IEEE TVCG (Proc. InfoVis), vol. 13, no. 6, pp. 1224-1231, 2007.

[82] H. Zhao, C. Plaisant, and B. Shneiderman, "I Hear the Pattern" Interactive Sonification of Geographical Data Patterns, Ext. Abs. CHI, pp. 1905-1908, 2005. 